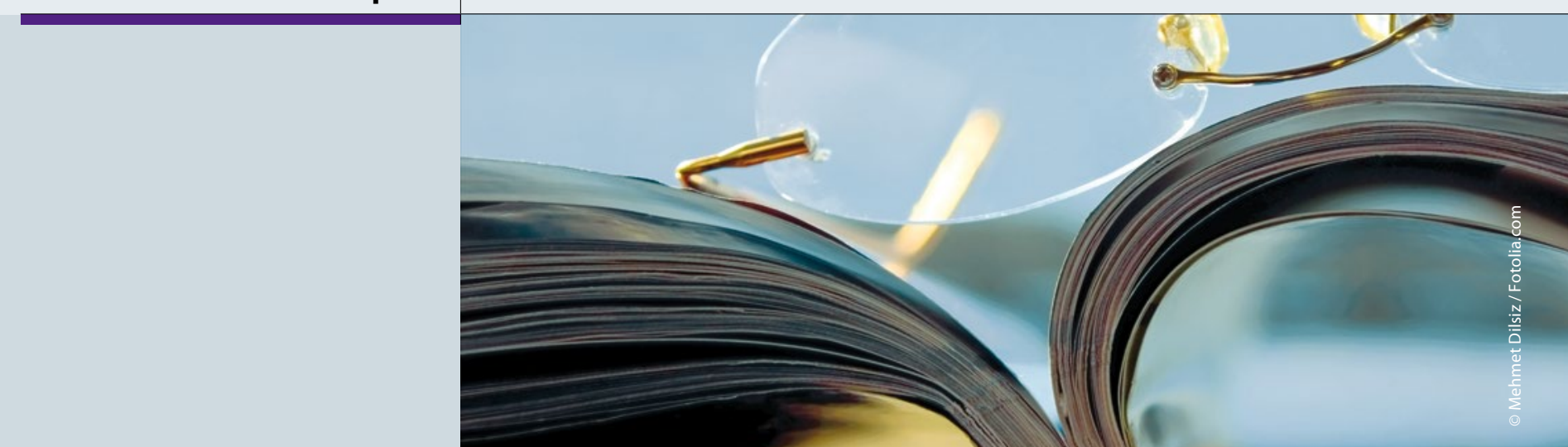

\section{Fehlbildungsrisiko durch die Pille?}

\section{Frauen, die kurz vor oder sogar noch zu Beginn einer Schwangerschaft die Pille genommen haben, müssen offenbar nicht befürchten, damit ihrem Kind geschadet zu haben.}

r rotz etwa 99\%iger Sicherheit der oralen Kontrazeption werden circa 9\% der Frauen im ersten Jahr der Anwendung schwanger. Weitaus mehr Frauen beenden die Einnahme der Pille zwar vor Eintritt der Schwangerschaft, bei ihnen vergeht zwischen beiden Ereignissen aber nur wenig Zeit. In beiden Situationen kann der Fetus unbeabsichtigt exogenen Sexualhormonen ausgesetzt werden, was in älteren Beobachtungsstudien mit einem erhöhten Risiko für bestimmte Fehlbildungen in Verbindung gebracht wurde. Biologisch erklärbar wäre dies, weil die zugeführten Hormone zum Beispiel Vitamin A-Serumspie- gel erhöhen oder die Folsäurekonzentration reduzieren können. Entwarnung signalisiert nun eine große Registerstudie.

Für die Studie haben Ärzte die prospektiv erhobenen Daten von 880.694 Lebendgeburten ausgewertet, die zwischen 1997 und 2011 in Dänemark registriert wurden. Bei 2,5\% der Kinder waren bis zum ersten Lebensjahr angeborene Anomalien entdeckt worden. Von den Müttern hatten $69 \%$ eine orale Kontrazeption mehr als drei Monate vor der Schwangerschaft beendet (Referenzgruppe) und $21 \%$ hatten nie die Pille genommen. Bei $8 \%$ der Mütter lagen zwischen Absetzen der Pille und Schwangerschaft weniger

\section{Hier steht eine Anzeige.}

$$
\text { 를 Springer }
$$

Einnahme oraler Kontrazeptiva bis zum Eintritt der Schwangerschaft: Ob dadurch kindliche Missbildungen drohen, wurde in einer aktuellen dänischen Studie untersucht. als drei Monate. $1 \%$ der Frauen hatte die Einnahme unwissentlich noch nach dem Eintritt der Schwangerschaft fortgesetzt. Die Prävalenz von Fehlbildungen in den vier Gruppen betrug: 25,0, $25,1,24,9$ und 24,8 , jeweils pro $1.000 \mathrm{Ge}$ burten. Das Risiko für angeborene Anomalien war damit bei Frauen, die in zeitlicher Nähe zur Schwangerschaft die Pille genommen hatten, nicht erhöht auch dann nicht, wenn andere Einflüsse wie Alter und Rauchverhalten berücksichtigt wurden.

Die gezielte Analyse spezieller Fehlbildungen förderte ebenfalls keine Unterschiede zwischen den Gruppen zutage. Auch Bauchspalten, hypoplastische Linksherzsyndrome, Extremitätendefekte und Harnwegsanomalien - Fehlbildungen, die in früheren Studien zum Teil bei Kindern von Pillenanwenderinnen vermehrt beobachtet worden waren waren in der dänischen Kohorte nicht mit einer Pilleneinnahme in der Zeit um den Eintritt der Schwangerschaft assoziiert.

Ein leicht erhöhtes Risiko für Fehlbildungen wurde in der Studie überraschenderweise bei Frauen entdeckt, die nie orale Verhütungsmittel angewendet hatten. Die Studienautoren vermuten allerdings, dass dabei eine statistische Verzerrung im Spiel ist. Beispielsweise könnten dieser Gruppe vermehrt stark übergewichtige Frauen angehören - Adipositas ist eine Kontraindikation gegen eine orale Kontrazeption, aber auch ein Risikofaktor für Fehlbildungen.

Dr. Beate Schumacher

Charlton B M et al. Maternal use of oral contraceptives and risk of birth defects in Denmark: prospective, nationwide cohort study. BMJ 2016;352:h6712 